\title{
CONHECIMENTOS DOS ENFERMEIROS DE UNIDADES BÁSICAS DE SAÚDE SOBRE A SÍNDROME PÓS-POLIOMIELITE
}

Nadia Shirley Correia Sobral Soares ${ }^{1}$, Flavia Nunes Ferreira de Araujo ${ }^{1}$, Maria Janine Pereira Fernandes ${ }^{1}$, Maria Zélia Araujo ${ }^{1}$, Francisca Martins Silva ${ }^{1}$, Bárbara Jeane Pinto Chaves ${ }^{1}$

Objetivo: Verificar o conhecimento dos enfermeiros sobre a Síndrome pós-póliomielite em unidades básicas de saúde. Metodologia: Estudo descritivo, explicativo de campo com abordagem quanti-qualitativa, desenvolvido em setembro e outubro de 2017 , com 11 profissionais de unidades básicas de saúde. Utilizou-se instrumento semiestruturado com questões de abordagem relativa ao tema. Os resultados foram analisados pelo programa Microsoft Excel (2010) e analise de conteúdo de Bardin, e representados em gráficos e tabelas. Todo o percurso metodológico respeitou os princípios éticos de pesquisa com seres humanos. Resultados: Constatou-se como faixa etária mais prevalente dos profissionais entre 20 a 35 anos(72,73\%), 90,91\% eram do sexo feminino, 63,64\% possuiam pós graduação Latu Sensu e 63,64\% atuam na UBS entre seis e dez anos, $73 \%$ dos profissionais não conhecem a patologia. Conclusão: Identificou-se, deficiência de conhecimentos sobre a Síndrome pós-pólio, o que sinaliza necessidade de capacitação sobre a temática.

Descritores: Síndrome pós-poliomielite; Complicações; Cuidados de enfermagem.

\section{KNOWLEDGE OF NURSES FROM BASIC HEALTH UNITS ON POST-POLIOMIELITE SYNDROME}

Objective: To verify nurses' knowledge about post-poliomielite syndrome in basic health units. Methodology: Descriptive, explanatory field study with quantitative-qualitative approach, developed in September and October 2017, with 11 professionals from basic health units. A semistructured instrument was used with questions related to the topic. The results were analyzed by the program Microsoft Excel (2010) and analysis of content of Bardin, post and represented in graphs and tables. The whole methodological course respected the ethical principles of research with human beings. Results: The most prevalent age group was between 20 to 35 years old (72.73\%), 90.91\% were female, 63.64\% had a Latu Sensu postgraduate course and $63.64 \%$ worked in the UBS between six and ten years, $73 \%$ of the professionals do not know the pathology. Conclusion: We identified a lack of knowledge about post-polio Syndrome among professionals, which indicates a need for training on the subject.

Descriptors: Post-poliomielite syndrome; Complications; Nursing care.

\section{CONOCIMIENTOS DE LOS ENFERMEROS DE UNIDADES BÁSICAS DE SALUD SOBRE LA SÍNDROME POST-POLIOMIELITE}

Objetivo: Verificar el conocimiento de los enfermeros sobre el Síndrome post-poliomielite en unidades básicas de salud. Metodología: Estudio descriptivo, explicativo de campo con abordaje cuantitativo, desarrollado en septiembre y octubre de 2017, con 11 profesionales de unidades básicas de salud. Se utilizó un instrumento semiestructurado con cuestiones de abordaje relativas al tema. Los resultados fueron analizados por el programa Microsoft Excel (2010) y análisis de contenido de Bardin, poste y representados en gráficos y tablas. Todo el recorrido metodológico respetó los principios éticos de investigación con seres humanos. Resultados: Se constató como grupo de edad más prevalente de los profesionales entre 20 a 35 años (72,73\%), 90,91\% eran del sexo femenino, 63,64\% poseian post graduación Latu Sensu y 63,64\% actúan en la UBS entre seis y diez años, el 73\% de los profesionales no conocen la patología. Conclusión: Se identificó, deficiencia de conocimientos sobre el Sindrome post-polio, lo que señala necesidad de capacitación sobre la temática.

Descriptores: Síndrome post-poliomielite; Complicaciones; Cuidados de enfermería. 


\section{INTRODUÇÃO}

A poliomielite paralítica é uma doença aguda causada pelo poliovirus, com relatos históricos na humanidade há milhares de anos. Em meados do século XX, com a implantação de um programa de vacinação eficaz, o número de novos casos reduziu consideravelmente. Dessa forma, o poliovírus selvagem foi eliminado do hemisfério ocidental, e o número de novos casos continua a cair no resto do mundo. No Brasil, os primeiros dados epidemiológicos sobre a poliomielite datam de 1910, e em 1994, a Organização Mundial de Saúde (OMS) declarou o Brasil como livre de pólioํ․

Mesmo nesse contexto, após um longo período de estabilidade após uma infecção pelo virus da poliomielite, pacientes com ou sem sequelas da doença podem desenvolver novas incapacidades com diversos sintomas, consistente com a sindrome pós-poliomielite (SPP) ${ }^{2}$.

A Síndrome pós-poliomielite é uma condição que afeta sobreviventes da poliomielite aguda (PAA), decorridos anos da recuperação de um ataque agudo inicial do vírus. Constase que quando há um desgaste nos neurônios motores acometidos pelo vírus, que é reativado em média 20 anos, após o indivíduo haver tido o contato direto oral-fecal, por alimentos e produtos contaminados, ocorre a manifestação $0^{3,4}$.

São características da síndrome 0 aparecimento de fraqueza muscular, depressão, fadiga, insônia, dores musculares e nas articulações, dor nos membros pela presença do frio, resultando em uma diminuição da capacidade funcional de membros e/ou no surgimento de novas incapacidades. Alguns pacientes desenvolvem, ainda, dificuldade de deglutição e respiração, podendo levar a um problema pulmonar grave ${ }^{2}$

Os novos sinais da doença aparecem preferencialmente em membros, de indivíduos com maior fraqueza motora. A instalação da SPP tem fator variável de acordo com a idade e a apresentação da doença aguda. Quanto maior a idade, maior a possibilidade de desenvolver esta sindrome ${ }^{4}$.

Na maioria dos casos o aparecimento dos sintomas é lento, com períodos de estabilização seguida de agravo do quadro clínico. Mudanças e alterações motoras e funcionais podem interferir na forma do próprio corpo e comprometer a manutenção da estética corporal. A piora do quadro clínico varia de acordo com cada indivíduo, mudando assim suas atividades diárias e a vida cotidiana ${ }^{5}$

Os profissionais precisam estar cientes dos agravos e danos que a SPP pode acarretar ao indivíduo como forma de intervir previamente. Uma vez que, muitos desses, por não conhecer especificamente o que é a síndrome, que pode ser confundida com outro tipo de doença, pode não oferecer um diagnóstico correto e/ou conseguintemente tardio, comprometendo assim ainda mais sua qualidade de vida dos acometidos $^{6}$.

Com base nessa problemática, o estudo teve por objetivo: verificar o conhecimento dos enfermeiros sobre a Síndrome pós-poliomielite em unidades básicas de saúde.

\section{MÉTODOLOGIA}

\section{Tipo de estudo}

Estudo descritivo, explicativo e no que diz respeito a classificação teve caráter quanti/qualitativa, uma vez que esses dois últimos aspectos favorecem ao pesquisador uma abordagem mais ampla, englobando da melhor maneira o objetivo elaborado na pesquisa.

\section{Participantes da pesquisa}

População de 11 dos enfermeiros atuantes das 12 UBS do município. Para isso, foram utilizados como critério de inclusão todos os enfermeiros que compõem as equipes de saúde dos referidos locais, que exerçam sua função há mais de 12 meses e que concordaram em participar do estudo, assinando o termo de consentimento livre e esclarecido (TCLE). Foram excluídos aqueles que estavam de licenças ou em afastamento por algum motivo.

\section{Local do estudo}

A pesquisa se desenvolveu com enfermeiros de 12 unidades básicas de saúde (UBS) no município de Alagoa Grande-PB, localizada no brejo paraibano. As UBS funcionam de segunda a sexta feira, nos turnos manhã e tarde, sendo 11 UBS na zona urbana e $\mathrm{Ol}$ na zona rural. Cada equipe trabalha com cobertura de área adstrita, funcionando com a composição mínima de um médico generalista, um enfermeiro, um odontólogo, um técnico de enfermagem e pelo menos seis agentes comunitários de saúde que desenvolvem suas atividades com base em cronograma elaborado e população adstrita.

\section{Coleta dos dados}

A pesquisa aconteceu nos meses de setembro e outubro de 2017. O instrumento de coleta de dados correspondeu a um formulário semiestruturado, contendo questões sobre a caracterização do perfil do profissional, a qual contempla faixa etária, sexo, estado civil, escolaridade, tempo de formação, tempo de trabalho na UBS e trabalho em outra instituição, bem como questões subjetivas relativas ao tema. $\mathrm{O}$ instrumento foi validado por meio de um pré-teste com dois profissionais de conhecimento técnico específico sobre o assunto.

$\mathrm{Na}$ ocasião da coleta de dados, foi esclarecido aos participantes, individualmente, por meio de uma leitura prévia e explicativa, o objetivo da pesquisa e o modo de 
preenchimento do mesmo. Ao concluir as respostas, cada ficha foi revisada, quanto a existência de questões não respondidas e logo finalizada a coleta, com a informação de que os resultados seriam divulgados aos participantes posteriormente.

\section{Procedimentos de análise dos dados}

Os dados quantitativos foram tabulados e transformados em gráficos pelo programa Microsoft Excel (2010) para melhor interpretação e visualização dos resultados obtidos. A análise de dados qualitativos foi realizada de acordo com a Análise de Conteúdo de Bardin (2011)

A técnica de análise de conteúdo se compõe de três grandes etapas: a primeira fase - pré-análise (organização); segunda fase - a exploração do material (etapa dos dados registrados) e terceira fase - o tratamento dos resultados e interpretação (classificação dos elementos e suas diferenças e semelhanças).

\section{Procedimentos éticos}

O projeto de pesquisa foi apreciado pelo Comitê de Ética em Pesquisa do CESED (Centro de Ensino Superior e Desenvolvimento) e aprovado sob número de CAAE 74799517.9.0000.5175.

Aos participantes foi garantido o sigilo das informações, uma vez que no próprio formulário não seria necessário a identificação do profissional. Os formulários foram codificados em El, E2, E3,...Ell, apenas para organização da tabulação dos dados.

\section{RESULTADOS}

A população estudada contou com 11 profissionais, já que um deles não alcancou o perfil seletivo para o estudo. Observou-se que (10)90,91\% eram do sexo feminino e a faixa etária mais prevalente dos profissionais é (8)72,73\%, de 20 a 35 anos. Quanto ao grau de escolaridade, (7)63,64\% tem pós graduação Latu Sensu e (7)63,64\% atuam na UBS entre seis e dez anos. Além disso, (7) 63,64\% trabalhavam em outra instituição.

Tabela 1: Dados Sociodemográficos dos Profissionais. Alagoa Grande-PB, 2017

\begin{tabular}{|c|c|c|}
\hline Dados & $\mathbf{N}$ & $\%$ \\
\hline \multicolumn{3}{|l|}{ Faixa Etária } \\
\hline $\begin{array}{l}20-35 \text { anos } \\
36-55 \text { anos }\end{array}$ & $\begin{array}{l}8 \\
3\end{array}$ & $\begin{array}{l}72,73 \% \\
27,27 \%\end{array}$ \\
\hline \multicolumn{3}{|l|}{ Sexo } \\
\hline Feminino & 10 & $90,91 \%$ \\
\hline Masculino & 1 & $9,09 \%$ \\
\hline
\end{tabular}

\begin{tabular}{|c|c|c|}
\hline Solteiro & 4 & $36,36 \%$ \\
\hline Casado & 7 & $63,64 \%$ \\
\hline \multicolumn{3}{|l|}{ Escolaridade } \\
\hline Nivel Superior & 2 & $18,18 \%$ \\
\hline $\begin{array}{l}\text { Pós-graduação: Latu Sensu } \\
\text { Pós-graduação: Strictu Sensu }\end{array}$ & $\begin{array}{l}7 \\
2\end{array}$ & $63,64 \%$ \\
\hline \multicolumn{3}{|l|}{ Tempo de Formação } \\
\hline la 5 anos & 4 & $33,36 \%$ \\
\hline 6 a 10 anos & 7 & $63,64 \%$ \\
\hline \multicolumn{3}{|l|}{ Faixa Etária } \\
\hline $20-35$ anos & 8 & $72,73 \%$ \\
\hline $36-55$ anos & 3 & $27,27 \%$ \\
\hline \multicolumn{3}{|l|}{ Tempo de Trabalho na UBS } \\
\hline 1 a 5 anos & 4 & $33,36 \%$ \\
\hline 6 a 10 anos & 7 & $63,34 \%$ \\
\hline \multicolumn{3}{|l|}{ Trabalha em outra Instituição } \\
\hline Sim & 7 & $63,64 \%$ \\
\hline Não & 4 & $36,36 \%$ \\
\hline TOTAL & 11 & $100 \%$ \\
\hline
\end{tabular}

De acordo com o gráfico 1, (8)73\% dos profissionais não conhecem a patologia, os mesmos ainda afirmam não ter prestado assistência a nenhum portador. E, (7)64\% não sabem identificar os sinais e sintomas apresentados nos pacientes com a síndrome, (8)73\% não tem conhecimentos das possiveis complicações ocasionadas pela patologia e (10)91\% não tem conhecimento do tratamento.

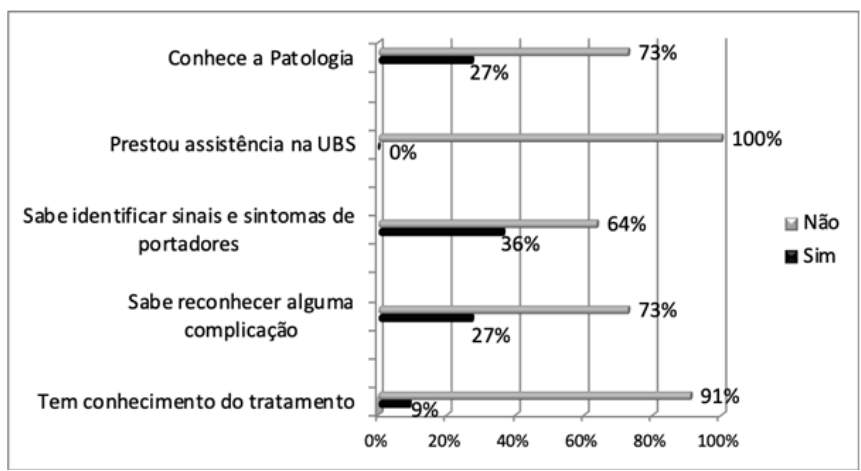

Gráfico 1- Informações referentes à Síndrome Pós-pólio. Alagoa Grande-PB, 2017

Diante das informações colhidas dos profissionais que trabalham nas UBS, (3)27\% conhecem a patologia e (4)36\% identificam alguns tipos de sinais e sintomas da SPP, percebendo-se, portanto, a deficiência de informações do grupo pesquisado. Por outro lado, aqueles que responderam "sim" também relataram quais sinais e sintomas: "Deficiência motora, dificuldade do desenvolvimento do tônus muscular"(El); "Deficiência física, tipo paralisia em um dos membros inferiores"(E2);"Febre alta; Paralisia flácida dos 
MMII, fraqueza muscular" (E3); "Dificuldades locomotoras, atraso nos marcos do desenvolvimento"(E4).

Diante da coleta realizada, (3) $27 \%$ sabe reconhecer alguma complicação. As principais complicações relatadas foram: "A Deficiência Física” (E2); "Paresia de MMII, Paraplegia de MMII, encurtamento de MMII”(E3); “Na puericultura: atraso nos marcos dos desenvolvimentos"(E4). Apenas (1)9\% dos profissionais têm conhecimento sobre o tratamento realizado ao portador da SPP, que foi citado a "fisioterapia motora" (E3).

Também foi investigado junto aos profissionais, quais as fontes que eles utilizam para obtenção de conhecimento sobre a temática em seu dia a dia. Alguns profissionais responderam mais de uma opção, obtendo-se o seguinte resultado: (5)45,45 $\%$ em artigos, (3)27,27\% em livros e, (4)36,36\% em nenhum meio de informação (Tabela 2).

Tabela 2- Fontes de conhecimento sobre a Sindrome Póspólio. Alagoa Grande-PB, 2017.

\begin{tabular}{|ccc|}
\hline $\begin{array}{c}\text { Fonte de } \\
\text { Conhecimento }\end{array}$ & Número de profissionais & $\%$ \\
\hline Livros & 03 & 27,27 \\
\hline Artigos & 05 & 45,45 \\
\hline Jornal & 01 & 9,09 \\
\hline Internet & 04 & 36,36 \\
\hline Redes Sociais & 01 & 9,09 \\
\hline Nenhum & 04 & 36,36 \\
\hline
\end{tabular}

Abusca incessante pelo enriquecimento do conhecimento, produz profissionais aptos a intervir satisfatoriamente, assim quando indagado junto aos profissionais pesquisados que estratégias podem ser realizadas na busca por mais conhecimentos sobre o que é a SPP, obteve-se:

"Capacitação dos profissionais através de educação permanente, cursos de reações adversas pós vacinal" (El); “Capacitação para os profissionais" (E2); “Atualização dos conhecimentos através das publicações do MS" (E3); "Sugiro que a pesquisadora explane seus conhecimentos com os entrevistados após o resultado das pesquisas coletadas, visto que a temática é pouco discutida e estudada" (E4); "Intervenções de educação continuada oferecidas pela gestão" (E5); "Campanhas da Secretaria Estadual e MS, para que assim nós profissionais possamos nos capacitar" (E6); "Palestras relacionadas ao tema abordado, livros, artigos entre outros" (E7); "Curso de aperfeiçoamento em vacina" (E8); "Mais capacitações sobre o tema, visto que a poliomielite é uma doença em erradicação no Brasil e casos são raríssimos" (E9); "Qualificação profissional/ educação permanente" (E10); "Inserção do tema nos conteúdo da atenção básica (manuseios, protocolos, cadernos, esclarecimentos na mídia)" (Ell).

\section{DISCUSSÃO}

A pesquisa evidenciou prevalência do sexo feminino entre os entrevistados, resultado semelhante a outra realizada com 37 profissionais de Enfermagem, na cidade de Caruaru, agreste pernambucano. É oportuno salientar que a maioria dos trabalhadores de enfermagem são mulheres, predominância característica dessa profissão7,8. Além disso, à maioria dos participantes dispõe de outro vínculo empregatício, o que, em muitas conjunturas, o trabalhador convive com a dupla jornada no intuito de melhorar as condições financeiras?

A respeito da patologia referida, embora crônicos, os efeitos tardios da poliomielite podem ser minimizados nos seus sintomas, mediante determinadas orientações. No entanto, percebe-se ser insuficiente esse conhecimento pela maior parte dos profissionais, o que limita a sua intervenção (10). Esse fato é corroborado com a pesquisa, quando mais da metade dos entrevistados relatam não saber identificar os sinais e sintomas apresentados pelos paciente, além dos mesmos ainda afirmarem não ter prestado assistência a nenhum portador, o que sinaliza para a possibilidade de existência de casos, porém confundidas com outras patologias com sintomatologias semelhantes.

Outro sim, quando a maioria não consegue identificar os sinais e sintomas apresentados nos pacientes, evidencia uma problemática bastante relevante. É importante que aqueles que fazem parte da assistência saibam caracteriza-los a partir do aparecimento de fraqueza muscular, depressão, fadiga, insônia, dores musculares e nas articulações, dor nos membros pela presença do frio, provocando uma redução da capacidade funcional de membros e/ou no surgimento de novas incapacidades, além de dificuldade de deglutição e respiração, como forma de intervir previamente, evitando assim situações mais graves ${ }^{11}$.

Quanto a desconhecem as possiveis complicações ocasionadas pela patologia, é preciso ressaltar que, embora possam ser confundidas, muitas vezes, com outras patologias, elas relacionam-se as mudanças e alterações motoras e funcionais que podem interferir na forma do próprio corpo e comprometer a manutenção da estética corporal. A piora do quadro clínico varia de acordo com cada indivíduo, mudando assim suas atividades diárias e a vida cotidiana ${ }^{5}$

Esses profissionais precisam saber também, que após a reativação do vírus, sintomas como febre e diarreia se apresentam, período em que começa a sua fase de ativação atingindo o sistema nervoso, o sistema gástrico e a corrente sanguínea, podendo levar vários danos ao indivíduo, chegando a afetar neurônios motores e acarretando sequelas como paralisia e atrofia dos membros ${ }^{1-4}$

Ainda sobre o estudo, quando se constatou que uma minoria tem conhecimento sobre o tratamento realizado ao 
portador da SPP, torna-se pertinente conhecer que alguns métodos como, terapia aquática e fisioterapias têm sido colocados em prática para atendimento dessas pessoas, produzindo grande êxito no cuidado de alguns casos, mesmo que com pouco avanço na melhora, porém funcionando como tratamentos paliativos, para que a tomada de decisões tornese positiva ${ }^{5}$.

No entanto, mesmo a pesquisa apontando para o desconhecimento dos profissionais sobre diversos pontos da temática, percebe-se interesse em preencher essa lacuna, já que a maioria afirma buscar informações em fontes de conhecimentos, especialmente em artigos científicos. Entretanto, quando para a composição e discussão do assunto, com busca de pesquisas recente, comprovouse limitados registros atuais e poucas pesquisas sobre a temática.

No que tange a necessidade de fomentar o conhecimento, os participantes da pesquisa também pontuaram sobre a importância de capacitações/educação permanente para seu auto aperfeiçoamento. Assim, a educação permanente é compreendida como a educação que se desenvolve no trabalho, pelo trabalho e para o trabalho. É uma das modalidades de educação no contexto laboral, a fim de dar respostas às transformações ocorridas no mundo do trabalho, deve ser considerada como uma estratégia para a qualificação dos trabalhadores ${ }^{13}$.

Em capacitações as informações dadas à equipe multiprofissional podem contribuir para melhor assistência às pessoas com a SPP. Assim, os profissionais estarão preparados para esclarecer sobre o problema e encaminhar aos serviços especializados. Para tanto, é necessário que os casos sejam mais bem investigados e avaliados ${ }^{12}$.

Em 2012, a poliomielite foi declarada uma emergência global, depois de serem registrados surtos considerados explosivos em países que, até então, estavam livres da doença e, como consequência, a SPP ${ }^{14}$

Diante disso, é de suma importância o conhecimento dos profissionais, no caso do estudo, os de enfermagem sobre a patologia como forma de identificar casos novos atendidos nas unidades que por vezes podem passar despercebidos ou confundidos com outras enfermidades, empregando assim um tratamento eficaz, promovendo assim uma melhor qualidade de vida a esses pacientes.

\section{Limitações do estudo}

Embora a pesquisa busque contribuições significativas para o cuidado de enfermagem, houve certa dificuldade em relação ao tempo disponível dos entrevistados devido a demanda de atendimento no serviço. No entanto, tal situação não impossibilitou que a mesma acontecesse satisfatoriamente.

\section{Contribuições para a prática}

Considerando a evidente necessidade dos profissionais de saúde em manter-se em constante processo de atualização, uma vez que a cada dia a assistência prestada ao paciente oferece novos desafios, espera-se que o estudo em questão possa oferecer subsídios ao fomentar o conhecimento dos sujeitos envolvidos no processo de cuidar, com vistas a promover uma excelente atuação na prática.

\section{CONCLUSÃO}

Diante os relatos obtidos da entrevista aos profissionais, percebe-se uma deficiência sobre o tema abordado, quanto ao conhecimento do que é a Sindrome pós-poliomielite, os sinais e sintomas, complicações e tratamentos adequados e específicos e meios que venham proporcionar uma melhor qualidade de vida às pessoas acometidas com a síndrome.

O aspecto positivo da pesquisa foi o relatado dos profissionais em buscar informações sobre o tema. Os profissionais demonstraram grande interesse sobre a SPP desde a imunização da poliomielite, as normas de adequação e armazenamento da vacina na Unidade Básica de Saúde, até as possiveis complicações que a doença ou a aplicação do imunobiológico podem ocasionar à criança.

Com isso, os profissionais necessitam de uma qualificação adequada, que seja em parceria com município, estados e governo federal, visto que a Síndrome é pouco debatida e divulgada. Os profissionais precisam estar aptos, qualificados a receber o paciente na rede pública com maior segurança, e poder encaminhá-lo ao serviço especializado.

\section{Contribuição:}

Concepção e/ou desenho, análise e interpretação dos dados, redação do artigo, revisão crítica, revisão final Nadia Shirley Correia Sobral Soares ,Flavia Nunes Ferreira de Araujo, Maria Janine Pereira Fernandes ,Maria Zélia Araujo, Francisca Martins Silva, Bárbara Jeane Pinto Chaves: 


\section{REFERÊNCIAS}

1. Quadros AAJ, Conde MTRP, Marin LF, Silva HCA, Silva TM, Paula BEM, et al. Frequency and clinical manifestations of post-poliomyelitis syndrome in a brazilian tertiary care center. Arq Neuro-Psiquiatr [Internet]. 2012 [cited 2017 Nov 29]: 70(8):571-3. Available from: http://www.scielo.br/ pdf/anp/v70n8/v70n8a02.pdf

2. Viana CF, Pradella-Hallinan M, Quadros AAJ, Marin LF, Oliveira ASB. Circadian variation of fatigue in both patients with paralytic poliomyelitis and post-polio syndrome. Arq Neuro-Psiquiatr [Internet]. 2013 [cited 2017 Nov 29]; 71(7):442-5. Available from: http://www.scielo.br/pdf/anp/v7ln7/ 0004-282X-anp-71-07-442.pdf

3. Garip Y, Eser F, Bodur H, Baskan B, Sivas F, Yilmaz O. Qualidade de vida relacionada com a saúde em sobreviventes turcos da pólio: impacto pós-pólio na saúde relacionada com a qualidade de vida em termos de estado funcional, gravidade da dor, fadiga e funcionamento social e emocional. Rev Bras Reumatol [Internet]. 2017 [cited 2017 Nov 29]; 57(1):1-7. Available from: http://www.scielo.br/pdf/rbr/v57nl/0482-5004-rbr-57-01-0001. pdf

4. Pereira RDB, Silva TM, Quadros AAJ, Orsini M, Schmidt B, Silva HCA, et al. Assessment of energy expenditure in individuals with post-poliomyelitis syndrome. Arq Neuro-Psiquiatr. [Internet]. 2017 [cited 2017 Nov 29]; 75(3):147-52. Available from: http://www.scielo.br/pdf/anp/v75n3/ 0004-282X-anp-75-03-0147.pdf

5. Lira CAB, Minozzo FCi, Sousa BS, Vancini RL, Andrade MS Quadros AAJ et al. Função pulmonar em pessoas com sindrome pós-poliomielite: um estudo transversal. J Bras Pneumol [Internet]. 2013 [cited 2017 Dec 06]; 39(4):455-60. Available from: http://www.scielo.br/pdf/jbpneu/v39n4/ 1806-3713-jbpneu-39-04-0455.pdf

6. Nascimento DR. As campanhas de vacinação contra a poliomielite no Brasil (1960-1990). Ciênc saúde coletiva [Internet]. 2011 [cited 2017 Dec 08]; 16(2):501-11. Available from: http://www.scielo.br/pdf/csc/vl6n2/ vl6n2al3.pdf

7. Aquino M, Barros LP, Brito AS, Ferreira EB, Medeiros SEG, Santos ER. Centro de material e esterilização: acidentes de trabalho e riscos ocupacionais. Rev SOBECC [Internet]. 2014 [cited 2016 Sep 12]:19(3):148-54. Available from: http://www.sobecc.org.br/ arquivos/artigos/2015/pdfs/ site_sobecc_v19n3/06_sobecc.pdf
8. Tanabe LP, Kobayashi RM. Perfil, competências e fluência digital dos enfermeiros do Programa de Aprimoramento Profissional. Rev Esc Enferm USP [Internet]. 2013 [cited 2016 Sep 18]:47(4):943-9. Available from: http:// www.scielo.br/pdf/reeusp/v47n4/en_0080-6234-reeusp-47-4-0943.pdf

9. Rocha MCP. Martino MMF Grassi-Kassisse DM, Souza AL. Stress among nurses: an examination of salivary cortisol levels on work and day off. Rev esc enferm USP [Internet]. 2013 Oct [cited 2017 Dec 21]: 47(5):1187-94. Available from: http://www.scielo.br/pdf/reeusp/v47n5/0080-6234-reeusp-47-05-1187.pdf

10. Orsini M, Kale JN. Mello MP. Machado D, Bastos VH, Joppert D, et al Gerenciamento da dor na Síndrome Pós-poliomielite: estudo de caso. Fisioter Pesqui [Internet]. 2011 [cited 2017 Dec 21]; 18(4):382-7. Available from: http://www.scielo.br/pdf/fp/v18n4/15.pdf

11. Ventura T. Poliomielite e seus efeitos tardios: viver ao ritmo da doença. Rev Port Med Geral Fam [Internet]. 2015 [cited 2017 Nov 29]: 31(5):326-33. Available from: http://www.scielo.mec.pt/pdf/rpmgf/v3ln5/v3ln5a06.pdf

12. De Biase NG, Moraes BT, Leb MDA, Korn GP, Padovani M, Moraes M, et al. Manifestações faringo-laringeas da sindrome pós-poliomielite. Arquivos Int Otorrinolaringol (Impr.) [Internet]. 2011 [cited 2017 Dec 21]; 15(4):52933. Available from: http://www.scielo.br/pdf/aio/v15n4/al9v15n4.pdf

13. Sade PM, Peres AM. Desenvolvimento de competências gerenciais do enfermeiro: diretriz para serviços de educação permanente. Rev esc enferm USP [Internet]. 2015 [cited 2017 Dec 08]; 49(6):988-94. Available from: http://www.scielo.br/pdf/reeusp/v49n6/0080-6234-reeusp-49-06-0991.pdf

14. Durante ALTC, Poz MRD. Saúde global e responsabilidade sanitária brasileira: o caso da erradicação da poliomielite. Saúde Debate [Internet]. 2014 [cited 2018 Apr 17]:38(100):129-38. Available from: http://dx.doi. org/10.5935/0103-104.20140007 